\title{
FTX contributes to cell proliferation and migration in lung adenocarcinoma via targeting miR-335-5p/NUCB2 axis
}

\author{
Xiaodong Huo ${ }^{1 \dagger}$, Huixing Wang ${ }^{2 \dagger}$, Bin Huo ${ }^{1}$, Lei Wang ${ }^{1}, \mathrm{Kuo} \mathrm{Yang}^{3}$, Jinhuan Wang ${ }^{1}$, Lili Wang ${ }^{1}$ \\ and Haitao Wang ${ }^{1 *}$ (1)
}

\begin{abstract}
Background: Extensive studies revealed that long non-coding RNAs (IncRNAs) could act as a regulator in tumors, including lung adenocarcinoma (LUAD). LncRNA FTX transcript, XIST regulator (FTX) has been reported to regulate the biological behaviors of some cancers. Nevertheless, its functional role and molecular mechanism remain obscure in LUAD. Our current study concentrates on exploring the biological function of FTX in LUAD.

Methods: RT-qPCR was used to test the expression of FTX, miR-335-5p or NUCB2 in LUAD cells. The effect of FTX on LUAD progression was investigated by colony formation, EdU, flow cytometry, TUNEL, transwell and western blot assays. The interaction between microRNA-335-5p (miR-335-5p) and FTX or nucleobindin 2 (NUCB2) was confirmed by luciferase reporter assay.

Results: RT-qPCR showed that FTX expression was up-regulated in LUAD cell lines. Loss-of-function assay indicated that FTX accelerated cell proliferation, migration and invasion, while inhibited cell apoptosis in LUAD. Besides, miR335-5p, lowly expressed in LUAD cells, was discovered to be sponged by FTX. Subsequently, NUCB2 was identified as a target gene of miR-335-5p. Additionally, it was confirmed that NUCB2 functioned as an oncogene in LUAD. Rescue assays indicated that LUAD progression inhibited by FTX knockdown could be restored by NUCB2 up-regulation.
\end{abstract}

Conclusion: FTX played an oncogenic role in LUAD and contributed to cancer development via targeting miR335-5p/NUCB2 axis.

Keywords: FTX, miR-335-5p, NUCB2, Lung adenocarcinoma

\section{Background}

Lung cancer is one of the most aggressive tumors and its incidence and mortality demonstrated a swift growth in recent decades [1]. It can be histologically divided into small cell lung carcinoma (SCLC) and non-small cell lung carcinoma (NSCLC). As a predominant type of NSCLC, lung adenocarcinoma (LUAD) accounts for a quite

\footnotetext{
*Correspondence: peterrock@126.com

${ }^{\dagger}$ Xiaodong Huo and Huixing Wang-Co-first authors

${ }^{1}$ Department of Oncology, The Second Hospital of Tianjin Medical University, No. 23 Pingjiang Road, Hexi District, Tianjin 300211, China

Full list of author information is available at the end of the article
}

important part of total lung cancer cases [2]. Although medical level has been improved, the clinical therapy of LUAD is still unsatisfactory because of late diagnosis, metastasis and recurrence [3]. Therefore, it is necessary to explore the potential biomarkers and molecular mechanism underlying LUAD for the improvement of the prognosis of LUAD patients.

Long noncoding RNAs (lncRNAs) are a group of RNAs longer than 200 nucleotides and have a limit in encoding proteins [4]. Substantial reports uncovered that abnormally-expressed lncRNAs are implicated in cancer occurrence and progression [5, 6]. For example,

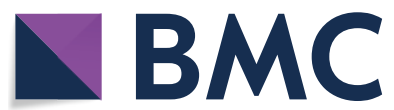

(c) The Author(s) 2020. This article is licensed under a Creative Commons Attribution 4.0 International License, which permits use, sharing, adaptation, distribution and reproduction in any medium or format, as long as you give appropriate credit to the original author(s) and the source, provide a link to the Creative Commons licence, and indicate if changes were made. The images or other third party material in this article are included in the article's Creative Commons licence, unless indicated otherwise in a credit line to the material. If material is not included in the article's Creative Commons licence and your intended use is not permitted by statutory regulation or exceeds the permitted use, you will need to obtain permission directly from the copyright holder. To view a copy of this licence, visit http://creativeco mmons.org/licenses/by/4.0/. The Creative Commons Public Domain Dedication waiver (http://creativecommons.org/publicdomain/ zero/1.0/) applies to the data made available in this article, unless otherwise stated in a credit line to the data. 
lncRNA DANCR is up-regulated in nasopharyngeal carcinoma and promotes metastasis through the interaction of NF90/NF45 complex [7]. LncRNA SNHG8 presents a high level, accelerates tumorigenesis and predicts tumor recurrence in hepatocellular carcinoma [8]. Previous studies showed that lncRNA could function as a competing endogenous RNA (ceRNA) and affect cancer progression by sponging microRNAs (miRNAs) to release messenger RNAs (mRNAs) at post-transcriptional level $[9,10]$. For instance, lncRNA C5orf66-AS1 acts as a ceRNA in cervical cancer and promotes cancer cell proliferation by targeting miR637/RING1 axis [11]. LncRNA CDKN2BAS serves as a ceRNA in hepatocellular carcinoma and predicts poor prognosis via regulating miR-153-5p/ARHGAP18 axis [12].

FTX, locating at X-chromosome inactivation (XCI) center, is a highly conserved lncRNA and exerts important role in the biological process of cancers [13]. FTX possesses an X-inactive-specific transcript and acts as the chief regulator of $\mathrm{X}$-inactivation initiation. In addition, FTX was reported as a ceRNA and played oncogenic role in colorectal cancer [14] and gliomas [13]. Nevertheless, its function and regulatory mechanism have not been revealed in LUAD.

In this research, we probed the role and mechanism of IncRNA FTX in LUAD, and found that FTX was overexpressed in LUAD cells and contributed to LUAD progression by sequestering miR-335-5p and up-regulating NUCB2. This discovery provided a helpful theoretic basis for the exploration of LUAD therapeutic strategies.

\section{Materials and methods Cell lines}

Human LUAD cell line (H1650, H1975, A549 and H1299) and human normal lung epithelial cell line (BEAS-2B) used in this study were propagated in the DMEM culture medium under $5 \% \mathrm{CO}_{2}$ at $37{ }^{\circ} \mathrm{C}$. The $10 \% \mathrm{FBS}$ (Gibco, Waltham, MA) and $1 \%$ Pen/Strep solution were served as the supplements for DMEM. All cell lines were procured from the ATCC (Manassas, VA).

\section{RNA extraction and RT-qPCR}

Total RNAs from H1650 and H1975 cells were obtained with the help of TRIZOL reagent (Invitrogen, Carlsbad, $\mathrm{CA}$ ), and then converted into cDNA employing the PrimeScript RT reagent kit (Takara Bio, Tokyo, Japan). RTqPCR procedure was achieved using SYBR Green PCR Master Mix (Takara), followed by $2^{-\triangle \Delta C T}$ method analysis. Gene expression was relative to GAPDH or U6. The primers were shown in Table 1.

\section{Transfection}

The shRNAs specifically against FTX and NUCB2, as well as the pcDNA3.1/FTX and pcDNA3.1/NUCB2, were all produced by Genepharma (Shanghai, China). The negative control (NC) nonspecific shRNAs and empty pcDNA3.1 vectors were also acquired. In addition, miR335-5p mimics/inhibitor and NC mimics/inhibitor were synthesized by Genechem (Shanghai, China). Cell transfection was implemented by application of the Lipofectamine 2000 (Invitrogen) for $48 \mathrm{~h}$. The sequences were illustrated in Table 1.

\section{Colony formation}

Clonogenic LUAD cells were cultivated for 14 days in the 6-well plates at 500 cells per well, and then treated with the $0.5 \%$ crystal violet solution for staining in $4 \%$ paraformaldehyde. Finally, colonies were counted manually.

\section{EdU incorporation assay}

For EdU analysis, LUAD cells were cultivated for $3 \mathrm{~h}$ with the $100 \mathrm{ml}$ of EdU incorporation assay kit (Ribobio, Guangzhou, China), and then treated with $4 \%$ paraformaldehyde for fixation. After permeabilization, DAPI solution was used to counterstain cell nuclei. Proliferative cells were determined under fluorescent microscope (Leica, Wetzlar, Germany).

\section{Cell cycle analysis}

Cells with $48 \mathrm{~h}$ of transfection were collected, and then eluted with cold PBS. Fixed with $70 \%$ ethanol, transfected cells were treated with RNase A $(100 \mu \mathrm{g} / \mathrm{ml})$, followed by a staining of the propidium iodide solution. Incubated for 20 min, flow cytometry (BD Biosciences, San Jose, CA) was used to determine cell cycle distribution.

\section{Flow cytometer for apoptosis}

Transfected LUAD cells were harvested for $15 \mathrm{~min}$ of double-staining with the Annexin V FITC/PI detection kit (Invitrogen) in the dark at $4{ }^{\circ} \mathrm{C}$. The apoptotic rate of LUAD cells was determined by flow cytometer (BD Biosciences).

\section{TUNEL assay}

Cell apoptosis was also detected by TUNEL assay in line with the protocol of One-Step TUNEL Apoptosis Assay Kit (Beyotime, Shanghai, China). After fixation and permeabilization, cells were treated with the DAPI solution, apoptotic cells were observed under fluorescent microscope.

\section{Transwell assays}

The Matrigel-coated transwell chamber was procured from Corning Co (Corning, NY) for cell invasion analysis, 
Table 1 The sequences for the PCR primers of genes

\begin{tabular}{|c|c|c|}
\hline \multicolumn{3}{|l|}{ PCR primers } \\
\hline Gene name & Forward primers & Reverse primers \\
\hline FTX & GCCCATAGTGCTTCACTGGT & CATCCTTGCCTCAGAGGGA \\
\hline miR-335-5p & TCAAGAGCAATAACGAAAAATGTG & CTCTACAGCTATATTGCCAGCCAC \\
\hline NUCB2 & GAACGTGTTACGAGTCAGTTTTTAAT & GACAACTGAGATCCAGAGAGGTAAG \\
\hline U6 & CTCGCTTCGGCAGCACA & AACGCTTCACGAATTTGCGT \\
\hline GAPDH & TCCCATCACCATCTTCCA & CATCACGCCACAGTTTTCC \\
\hline \multicolumn{3}{|c|}{ shRNA sequence } \\
\hline sh-NC & \multicolumn{2}{|c|}{ CCGGCAGACATGATATCACATAGCTCTCGAGAGCTATGTGATATCATGTCTGTTTTTG } \\
\hline sh-FTX\#1 & \multicolumn{2}{|c|}{ CCGGCACTACATCTGGCTTACACTACTCGAGTAGTGTAAGCCAGATGTAGTGTTTTTTG } \\
\hline sh-FTX\#2 & \multicolumn{2}{|c|}{ CCGGGACGTATACAGAATACTTCGTCTCGAGACGAAGTATTCTGTATACGTCTTTTTG } \\
\hline sh-NC & \multicolumn{2}{|c|}{ CCGGGAGTCAGTGTATAGTGCATAGCTCGAGCTATGCACTATACACTGACTCTTTTTG } \\
\hline sh-NUCB2 & \multicolumn{2}{|c|}{ CCGGAACGTGTTACGAGTCAGTTTTCTCGAGAAAACTGACTCGTAACACGTTTTTTTG } \\
\hline
\end{tabular}

while cell migration was analyzed without Matrigel coating. $5 \times 10^{3}$ cells were placed into the upper chamber, while complete culture medium was added into the lower chamber. Invasive or migratory cells were fixed and stained with crystal violet $24 \mathrm{~h}$ later, and then counted under the microscope.

\section{In vivo assay and pulmonary metastasis assay}

Male BALB/c nude mice (4-week-old) purchased from the Second Hospital of Tianjin Medical University were used for animal experiments with the ethical approval of the the Second Hospital of Tianjin Medical University. The $\mathrm{BALB} / \mathrm{C}$ nude mice (five mice in a group) housed in a standard environment were subcutaneously inoculated with shFTX or sh-NC transfected H1650 cells $\left(1 \times 10^{6} /\right.$ mice $)$. Then, tumor growth was monitored every 4 days and the mice were sacrificed by cervical dislocation after 28 days. Later, the xenograft tumors were removed and weighed. Finally, RT-qPCR was employed for the detection of FTX expression and western blot was performed to measure NUCB2 protein level. For pulmonary metastasis assay, stably transfected H1650 cells were injected into the tail vein of mice. Two weeks later, mice were sacrificed and the lungs were dissected. Then, the metastatic nodules were counted.

\section{HE staining}

For HE staining, sections were deparaffinized and rehydrated, and then incubated with hematoxylin. After staining in acid ethanol and eosin with five dips, the sections were dehydrated and cleared. Finally, the images were obtained with a fluorescence microscope.

\section{Western blot}

The cellular total proteins were extracted from LUAD cells for treating with the $12 \%$ SDS-PAGE gel and shifting onto the PVDF membranes. After adding 5\% skimmed milk, the primary antibodies against the internal control GAPDH and slug, Vimentin, N-cadherin, E-cadherin, NUCB2, p-AKT, AKT, p-mTOR, mTOR, p-STAT3, STAT3 as well as the appropriate HRP-tagged secondary antibodies (all, Abcam, Cambridge, MA) were diluted and used for probing membranes. The blots were monitored by enhanced chemiluminescence reagent (Santa Cruz Biotechnology, Santa Cruz, CA).

\section{FISH}

The fixed LUAD cells were collected and air-dried for incubation in the hybridization buffer with the FTX FISH probe (Ribobio). Following adding the Hoechst staining solution, stained samples were assayed under fluorescent microscope.

\section{RNA pull down}

The cellular protein samples from LUAD cells were acquired in RIPA lysis buffer and incubated with the wild-type (WT) or mutated (Mut) biotinylated FTX probes for $1 \mathrm{~h}$ at $4{ }^{\circ} \mathrm{C}$. The streptavidin agarose magnetic beads were added to collect the pull-downs. The miRNA enrichments were analyzed by RT-qPCR.

\section{Dual-luciferase reporter assays}

Fragments of FTX or NUCB2 sequences which contained the miR-335-5p binding sites were amplified by RT-PCR and then cloned into pmirGLO reporter vectors (Promega, Madison, WI) to generate FTX-WT/NUCB2WT luciferase reporters. The mutations were constructed by Site-Directed Mutagenesis kit (Invitrogen) and named as FTX-Mut and NUCB2-/Mut. The recombinant reporter vectors were co-transfected into LUAD cells with indicated transfection plasmids for $48 \mathrm{~h}$, then 
Dual-Luciferase Reporter Assay System (Promega) was applied for luciferase activities.

\section{RNA immunoprecipitation (RIP)}

$1 \times 10^{7}$ LUAD cells were reaped from the RIP lysis buffer for cultivating with the beads-bound anti-Ago2 or antiIgG antibody (Millipore, Bedford, MA) in the RIP buffer. The RNA levels in the precipitates were estimated using RT-qPCR.

\section{Statistical analyses}

Results were presented as the mean \pm standard deviation (SD) of more than three independent bio-repeats. Data analysis in each group was processed by student's t-test or one-way analysis of variance (ANOVA) by application of PRISM 6 software (GraphPad, San Diego, CA). The p-value less than 0.05 was set as the threshold of significant level.

\section{Results \\ FTX was overexpressed and enhanced the progression in LUAD}

To explore the expression pattern of FTX in LUAD, we detected FTX expression in four LUAD cell lines (H1650, H1975, A549 and H1299). The human normal lung epithelial cell line (BEAS-2B) was seen as a control. As a result, FTX was highly expressed in LUAD cell lines, particularly in H1650 and H1975 cells (Fig. 1a), suggesting the aberrant expression of FTX in LUAD. Therefore, we explored the biological function of FTX in LUAD by designing loss-of-function assay. Before the experiment, FTX expression was stably silenced in H1650 and H1975 cells (Fig. 1b). It was well known that cell proliferation and apoptosis are the important processes involved in cancer progression. Therefore, the effect of FTX on LUAD cell proliferation and apoptosis was assessed. Colony formation assay and EdU assay manifested that FTX silencing notably lessened the LUAD proliferation of H1650 and H1975 cells (Fig. 1c, d). Further, cell cycle analysis showed that silenced FTX remarkably ascended the proportion of $\mathrm{H} 1650$ and $\mathrm{H} 1975$ cells in G0/G1 phases, and reduced cell proportion in $S$ and $G 2 / M$ phases (Additional file 1: Figure S1A). Later, we viewed that cell apoptosis was potently boosted in sh-FTX transfected H1650 and H1975 cells (Fig. 1e, f and Additional file 1: Figure S1B). Previous studies indicated that cancer metastasis was dependent on the abilities of cell migration and invasion [7, 15]. Based on this, we investigated the biological role of FTX in LUAD by testing migratory and invasive capacities of H1650 and H1975 cells. Results of transwell assay unveiled that knockdown of FTX conspicuously hindered cell migration and invasion in LUAD (Fig. 1g, h). Since epithelial-mesenchymal transition
(EMT) process is closely correlated with cell migration and invasion [16, 17], we evaluated whether FTX could induce EMT in LUAD cells. Western blot assay implied that protein level of epithelial marker (E-cadherin) was obviously ascended while protein levels of mesenchymal markers (N-cadherin and Vimentin) and EMT transcription factor (slug) were evidently alleviated in H1650 and H1975 cells transfected with sh-FTX (Fig. 1i). To be concluded, FTX acted as an oncogene in LUAD.

\section{FTX sponged miR-335-5p in LUAD}

Subsequently, we deciphered molecular mechanism that FTX involved in LUAD. It has been reported that lncRNA could regulate cancer progression by serving as miRNA sponge in the cytoplasm $[9,10]$. Thus, we detected the localization of FTX in LUAD cells by FISH assay, and results displayed that FTX was abundantly distributed in LUAD cell cytoplasm (Fig. 2a), giving the potential of ceRNA hypothesis. Using starBase (http://starbase.sysu. edu.cn), 6 miRNAs were predicted to possess complementary bases on the sequence of FTX. To further filtrate the selection, RNA pull down assay was performed. Results revealed that biotinylated wild-type FTX pulled down miR-335-5p while other miRNAs were not affected (Fig. 2b). Besides, the binding site between FTX and miR335-5p was projected and the mutation was constructed (Fig. 2c). Moreover, we found that miR-335-5p expressed at a low level in LUAD cells (Fig. 2d). To explore the relationship between miR-335-5p and FTX, we tested miR335-5p expression in sh-FTX transfected cells. Results disclosed that miR-335-5p expression was elevated upon FTX depletion (Fig. 2e). Then, the transfection efficiency of miR-335-5p mimics was examined. Results of RTqPCR displayed that miR-335-5p expression achieved an escalation by transfecting miR-335-5p mimics in LUAD cells (Fig. 2f). Interestingly, we found that the expression of FTX was not affected by up-regulated miR-335-5p (Fig. 2g). To further confirm the interaction between FTX and miR-335-5p, luciferase reporter assay was exploited. As we observed, the transfection of miR-335-5p mimics led to a decreased luciferase activity of FTX-WT vector while no evident changes were seen in FTX-Mut vector (Fig. 2h). Collectively, FTX acted as a sponge of miR335-5p in LUAD.

\section{MiR-335-5p down-regulation restored the influence of FTX knockdown on LUAD cells}

Here, we checked whether FTX boosted LUAD progression by sponging miR-335-5p. Hence, some rescue assays were designed and conducted. Firstly, the plasmid of miR-335-5p inhibitor was transfected into LUAD cells to repress miR-335-5p expression (Fig. 3a). In colony formation and EdU assays, miR-335-5p depletion could 


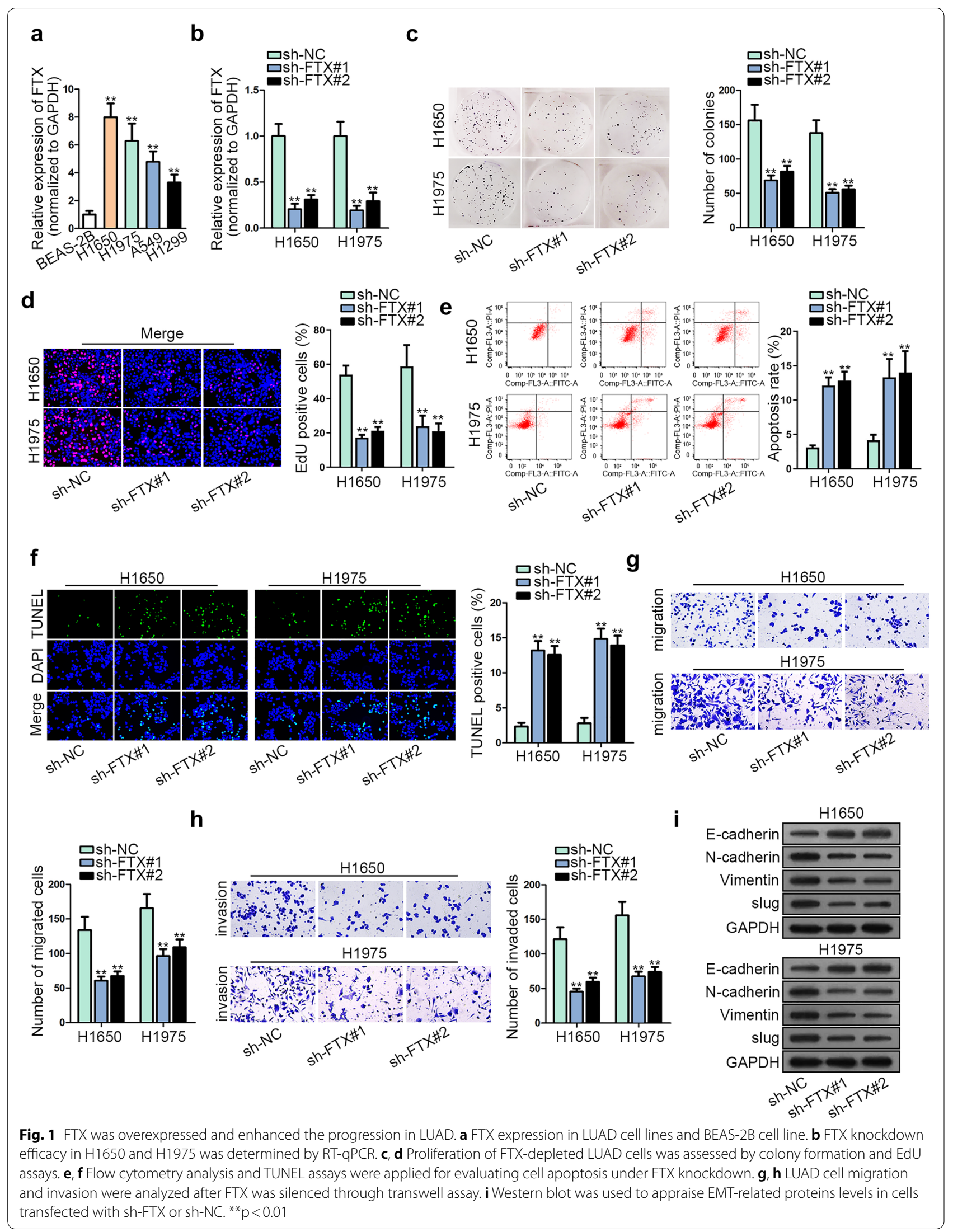




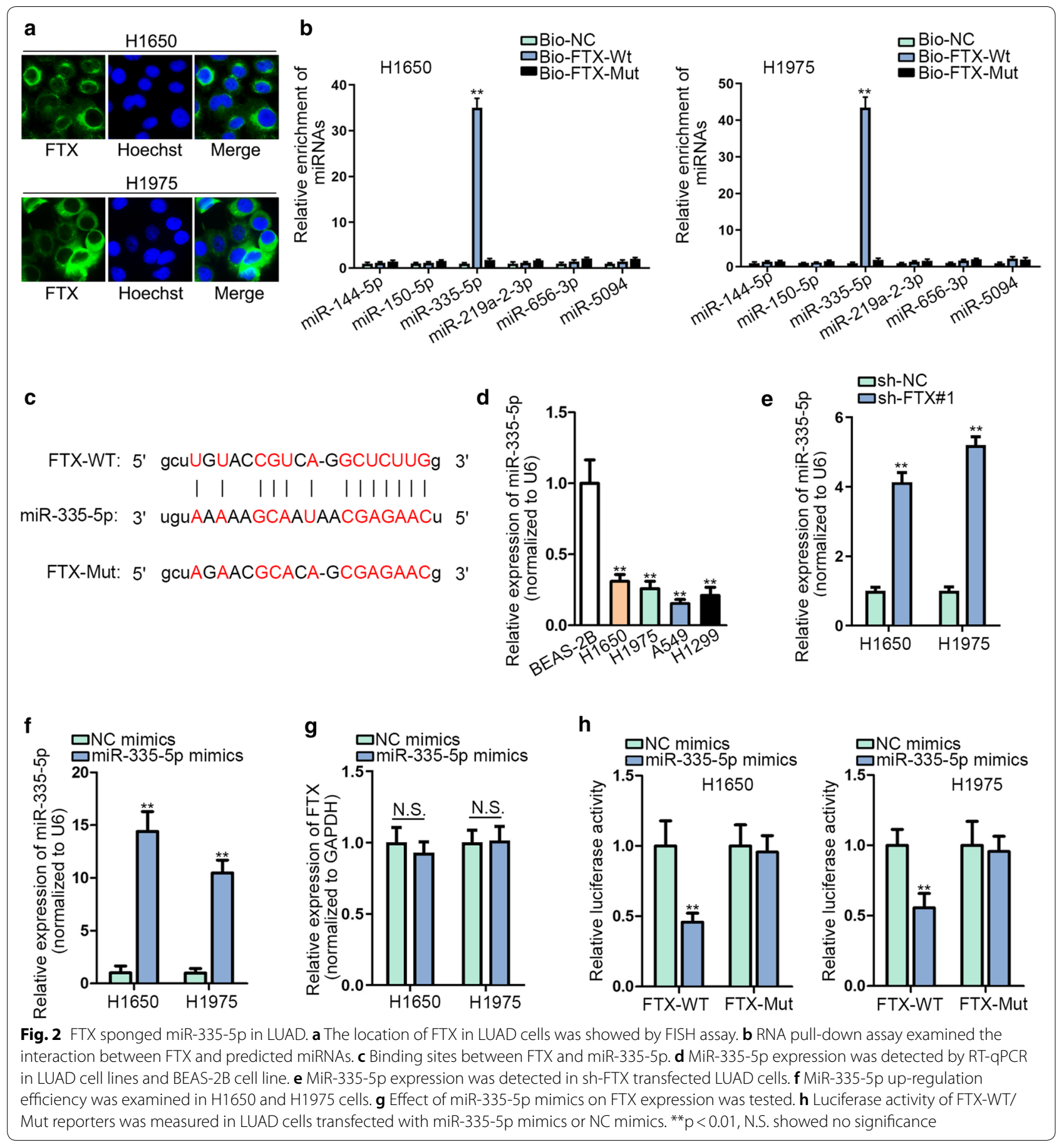

abrogate FTX knockdown-mediated suppression on LUAD cell proliferative capacity (Fig. 3b, c and Additional file 1: Figure S1C). In addition, the recovering effect of down-regulated miR-335-5p on the apoptosis increased by FTX down-regulation was seen in flow cytometry analysis and TUNEL assay (Fig. 3d, e and Additional file 1: Figure S1D). According to transwell assays, declined migratory and invasive abilities resulting from silenced FTX were abrogated by miR-335-5p repression (Fig. 3f, g). Data of western blot assay delineated that the effect of FTX deficiency on the protein levels of E-cadherin, N-cadherin, Vimentin and slug was countervailed by miR-335-5p suppression (Fig. 3h). To sum up, 


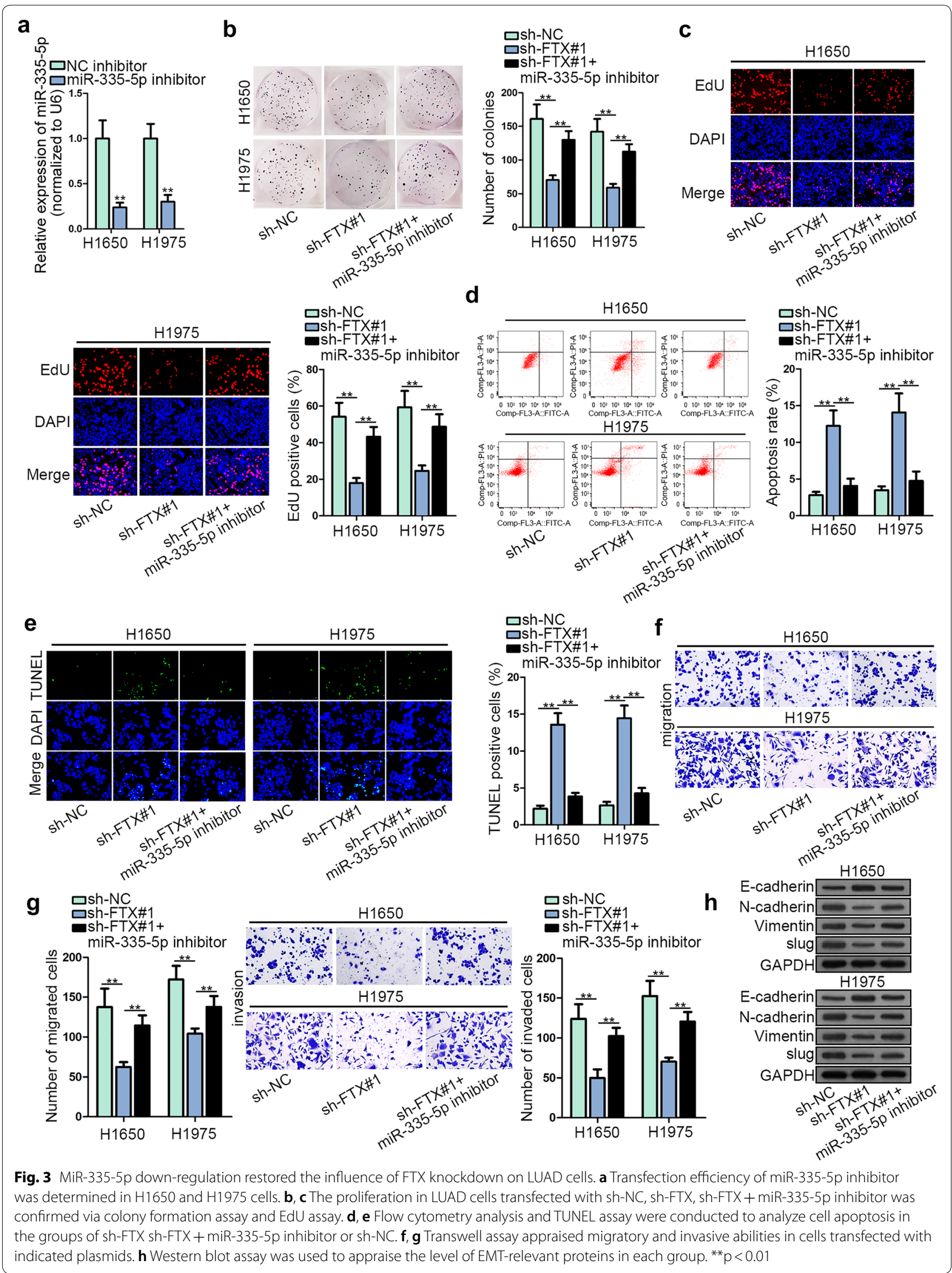


(See figure on next page.)

Fig. 4 NUCB2 was a target of miR-335-5p and acted as an oncogene in LUAD. a Effect of miR-335-5p mimics on the expressions of predicted mRNAs was validated through RT-qPCR. $\mathbf{b}$ MiR-335-5p was predicted to possess a binding site on NUCB2. $\mathbf{c}$ NUCB2 expression was assessed in LUAD cell lines and BEAS-2B cell line was seen as a control. $\mathbf{d}$ RIP assay testified the enrichment of FTX, miR-335-5p and NUCB2 in RISC. e Luciferase activity of NUCB2-WT/Mut in response to the indicated plasmids was certificated via luciferase reporter assay. $\mathbf{f}$ NUCB2 knockdown efficacy in H1650 and H1975 cells. $\mathbf{g}, \mathbf{h}$ The influence of sh-NUCB2 or sh-NC on LUAD cell proliferation was assessed by colony formation and EdU assays. i, $\mathbf{j}$ The apoptosis in sh-NUCB2 or sh-NC group was evaluated via flow cytometry analysis and TUNEL assay. $\mathbf{k}$, ITranswell assay was performed to appraise cell migration and invasion in sh-NUCB2 or sh-NC group. $\mathbf{m}$ Western blot assay was conducted to evaluate EMT-relevant protein levels in sh-NUCB2 or sh-NC group. ${ }^{* *} \mathrm{p}<0.01$

miR-335-5p down-regulation restored the influence of FTX knockdown on LUAD cell progression.

\section{NUCB2 was a target of miR-335-5p and acted as an oncogene in LUAD}

With the purpose of supporting ceRNA hypothesis, the targets of miR-335-5p were explored. Through starBase (an online tool), NPC2, LRRC8A and NUCB2 were predicted with the strict condition (pan-cancer $\geq 8$; degradome data $\geq 3$ and clip data $\geq 5$ ). To select the optimal gene for miR-335-5p in LUAD, the expressions of these mRNAs were measured in LUAD cells transfected with miR-335-5p mimics or NC mimics. As a result, NUCB2 expression was inhibited by up-regulated miR-335-5p while no apparent changes were observed in the other mRNAs (Fig. 4a). Besides, the complementary binding site between miR-335-5p and NUCB2 was predicted (Fig. 4b). Furthermore, NUCB2 was found at a high level in LUAD cell lines (Fig. 4c). It has been reported that Ago2 is the core component of RNA-induced silencing complex (RISC) and miRNAs could exert the effect of gene silencing by RISC [18]. To assess whether FTX, miR-335-5p and NUCB2 co-exist in the identical RISC, RIP assay was carried out. Results disclosed that FTX, miR-335-5p and NUCB2 were all abundant in the beads conjugated with Ago2 antibody but not IgG antibody (Fig. 4d). Later, it was exhibited in luciferase reporter assay that miR-335-5p mimics abated the luciferase activity of NUCB2-WT reporter rather than NUCB2-Mut reporter. In addition, the decreased luciferase activity was recovered by pcDNA3.1/FTX (Fig. 4e). Above results uncovered that NUCB2 was a target of miR335-5p in FTX-mediated ceRNA network. Subsequently, the functional role of NUCB2 in LUAD was assessed. The expression of NUCB2 was reduced in LUAD cells by transfecting sh-NUCB2 (Fig. 4f). We found that NUCB2 depletion weakened the proliferative ability of LUAD cells (Fig. 4g, h). Meanwhile, LUAD cell apoptosis was stimulated by sh-NUCB2 transfection (Fig. 4i, j and Additional file 1: Figure S1E). Moreover, cell migration, invasion and EMT were refrained upon NUCB2 knockdown (Fig. 4k-m). In addition, as we all known, many signals could control the above processes; thus, we explored the downstream signals of NUCB2. NUCB2 was reported to be associated with AKT/mTOR pathway and STAT3 pathway [19, 20]. As shown in Additional file 1: Figure S1F, FTX knockdown significantly decreased the levels of phosphorylated AKT and mTOR, and NUCB2 rescued the effect of silenced FTX. However, no detectable changes were observed in phosphorylated STAT3 and total levels of AKT, mTOR and STAT3, which suggested that NUCB2 could mediate AKT/mTOR rather than STAT3 pathway in LUAD. In summary, NUCB2 was a target of miR-335-5p and acted as an oncogene in LUAD.

\section{FTX promoted LUAD progression through increasing NUCB2 expression}

In order to further check the function of $\mathrm{FTX} / \mathrm{miR}$ 335-5p/NUCB2 axis in LUAD, rescue assays were designed and performed. At the beginning, we elevated NUCB2 expression by transfecting pcDNA3.1/NUCB2 in LUAD cells (Fig. 5a). Colony formation and EdU assays certified that down-regulation of FTX exceedingly curbed cell proliferation in LUAD while pcDNA3.1/ NUCB2 counteracted FTX silencing-imposed effect (Fig. 5b, c). Additionally, NUCB2 overexpression was also released G0/G1 arrest of LUAD cells caused by FTX depletion (Additional file 1: Figure S1G). In terms of LUAD cell apoptosis, FTX depletion-hastened apoptosis was neutralized by pcDNA3.1/NUCB2, as shown in flow cytometry analysis and TUNEL assay (Fig. 5d, e and Additional file 1: Figure S1H). The suppressed migratory and invasive abilities caused by down-regulation of FTX were recovered by overexpression of NUCB2 (Fig. 5f, g). Western blot assay pictured that escalation of E-cadherin protein level and decrease of N-cadherin, slug, and Vimentin protein levels caused by FTX knockdown could be counterbalanced by pcDNA3.1/NUCB2 (Fig. 5h). To summarize, FTX accelerated LUAD progression via lifting NUCB2 expression in vitro. Then, we investigated whether FTX played the same role in vivo. Nude mice were subcutaneously inoculated with H1650 cells stably transfected with sh-FTX or sh-NC. Images of tumors in sh-FTX and sh-NC groups were exhibited (Additional file 2: Figure S2A). Tumor volume and weight were respectively detected and evaluated. FTX knockdown 


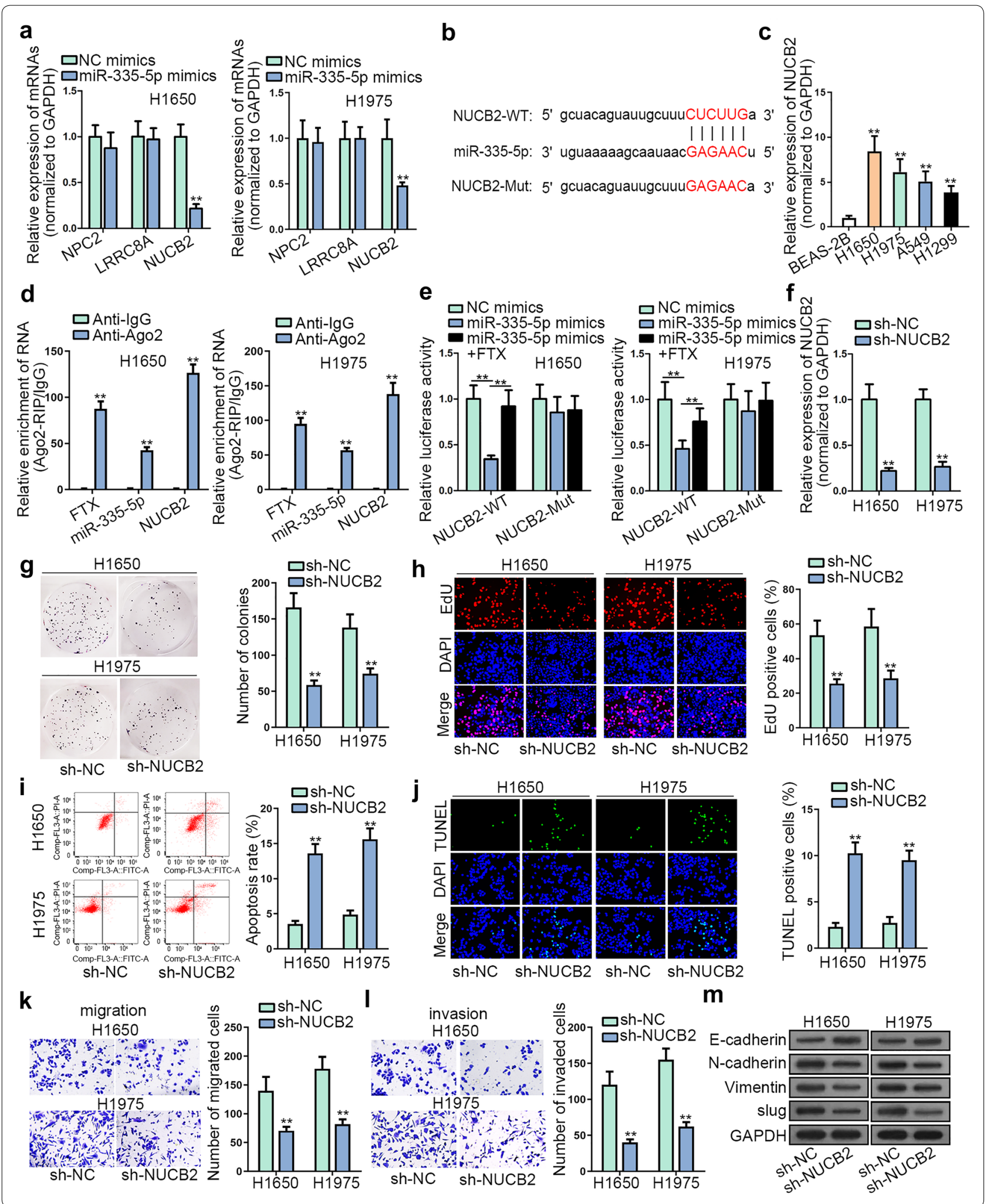



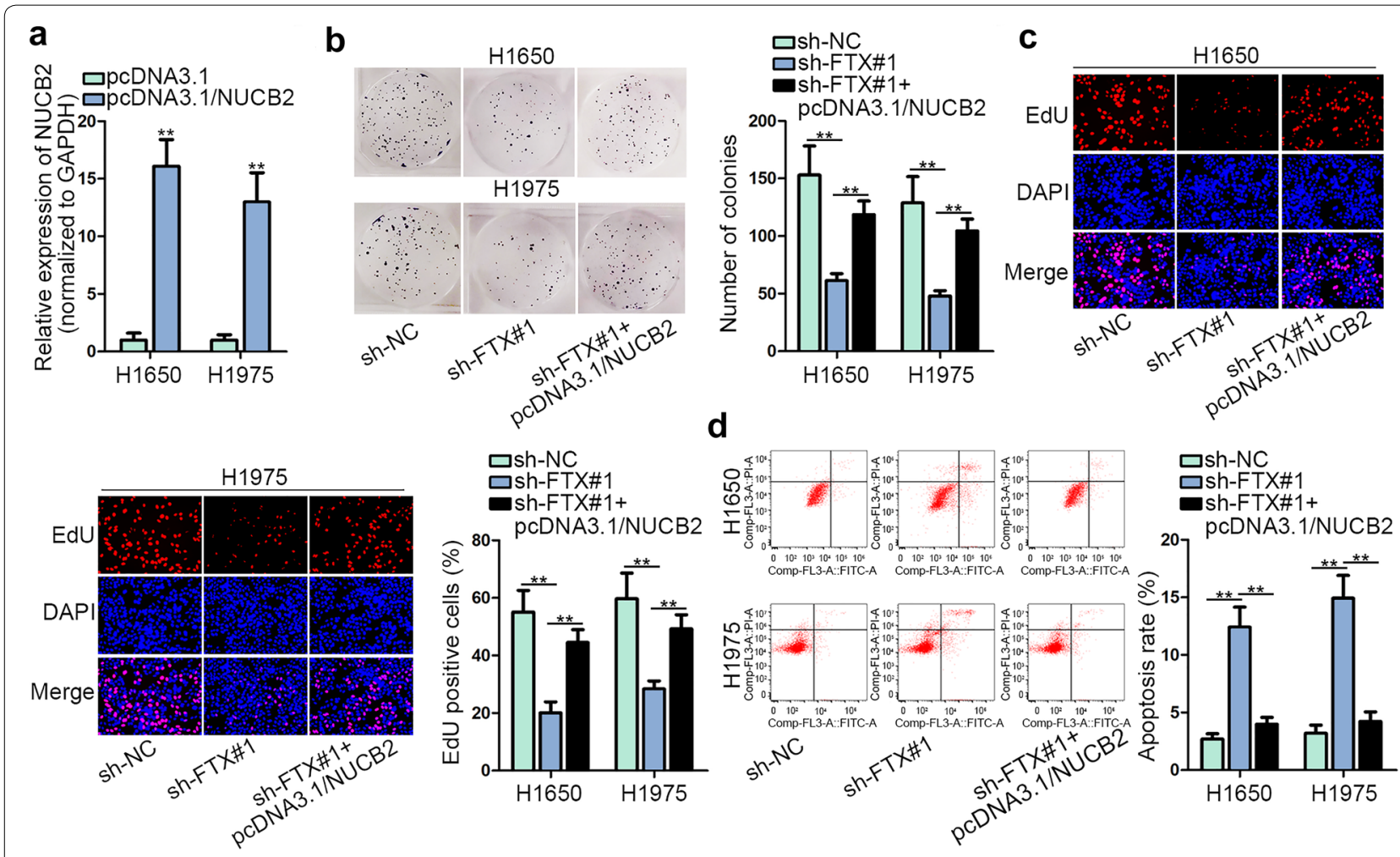

e
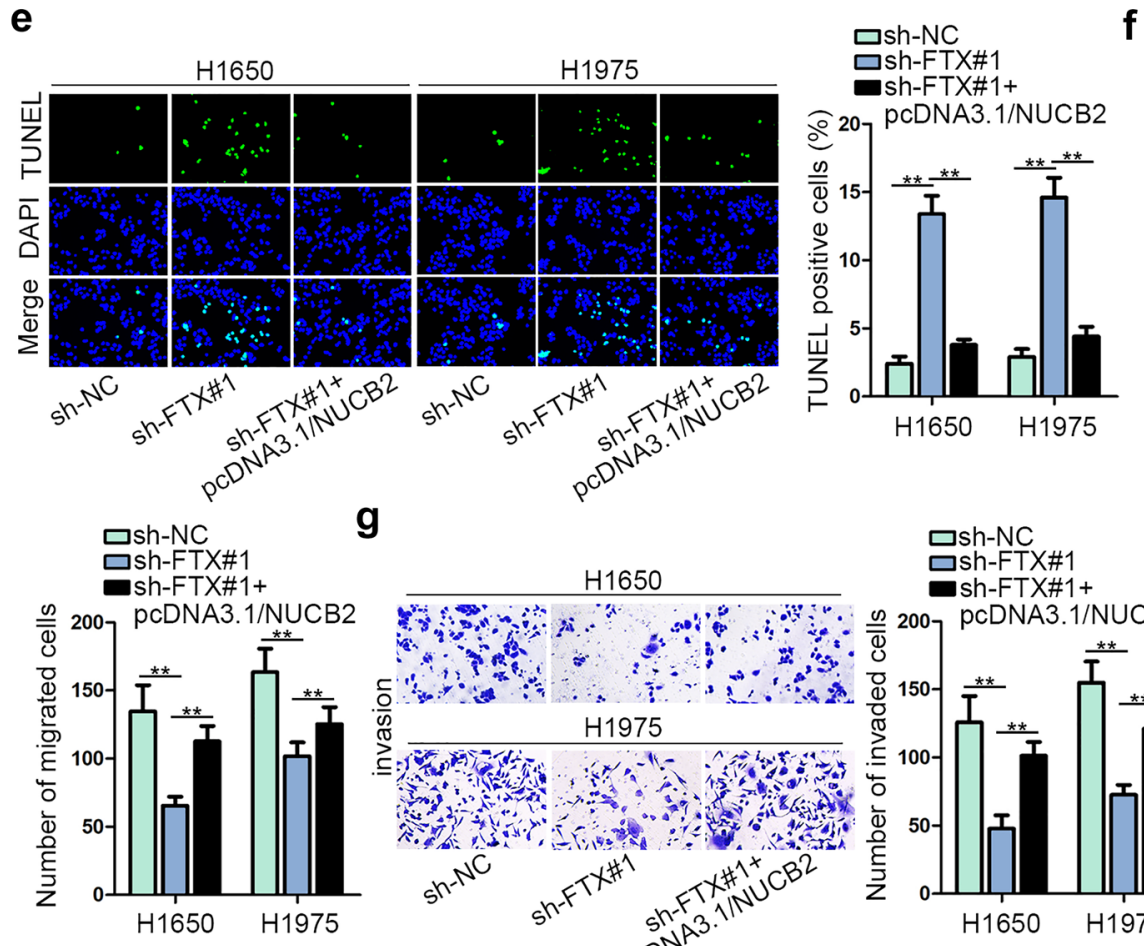

g
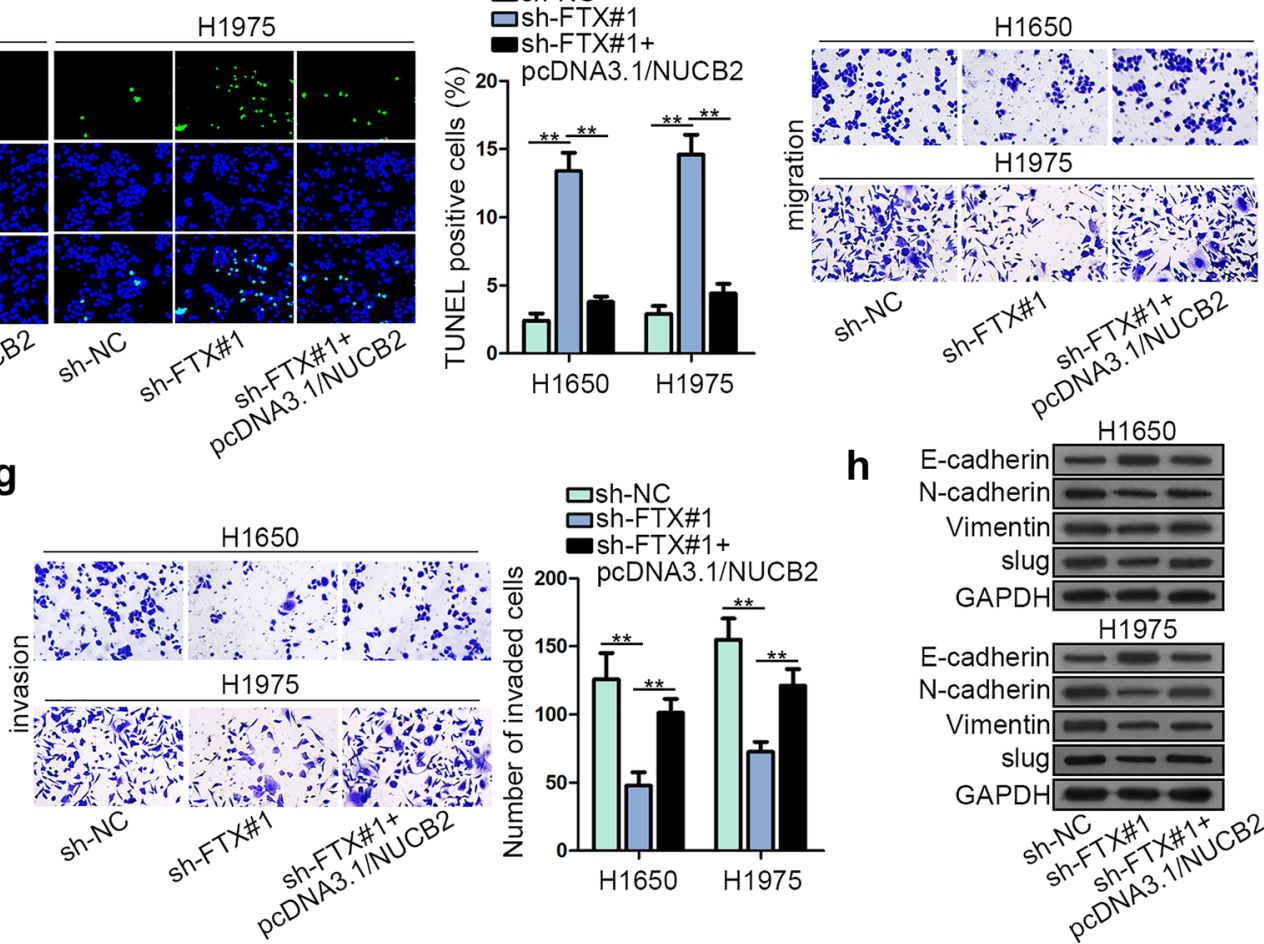

h

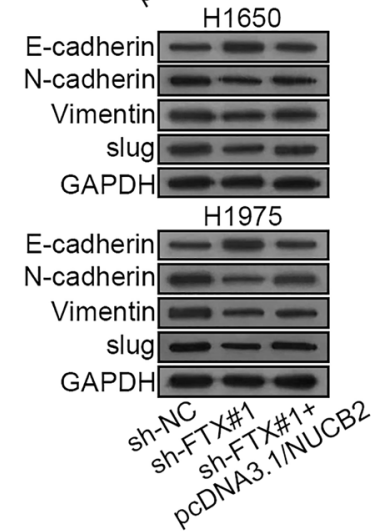

Fig. 5 FTX promoted LUAD progression through increasing NUCB2 expression. a Transfection efficiency of pCDNA3.1/NUCB2 was validated using RT-qPCR. b, c LUAD cell proliferation was verified in each group. $\mathbf{d}$, e The apoptosis of cells transfected with appointed plasmids was tested. $\mathbf{f}, \mathbf{g}$ The migration and invasion in cells transfected with the appointed plasmids were assessed. $\mathbf{h}$ EMT-related protein levels were detected with the transfection of indicated plasmids. ${ }^{* *} p<0.01$ 
reduced the volume and weight of tumors compared with sh-NC group (Additional file 2: Figure S2B, C). Then we assessed the expression of FTX and found that FTX expression was reduced due to FTX deficiency (Additional file 2: Figure S2D). Owing to the accelerative effect of FTX on cell migration and invasion, we determined the role of FTX deficiency in cell metastasis in vivo. Consistently, lung metastasis of mice was significantly hindered in sh-FTX group compared with sh-NC group (Additional file 2: Figure S2E). Finally, western blot assay examined that NUCB2 protein level was restrained under FTX silencing (Additional file 2: Figure S2F). Therefore, we concluded that FTX promoted LUAD progression through increasing NUCB expression.

\section{Discussion}

LUAD is an aggressive tumor with a high occurrence rate and mortality, and various dysregulated lncRNAs were reported to be associated with LUAD progression. For instance, lncRNA LIN28B-AS1 promotes the proliferation of LUAD cells [21]. LncRNA NEAT1 stimulates cell migration and hampers cell apoptosis in LUAD [22]. LncRNA SOX21-AS1 exacerbates cell proliferation and indicates a poor prognosis in LUAD [23]. LncRNA FTX was observed to be up-regulated in glioma and boost cell proliferation and invasion [13]. Nevertheless, it has not been studied in LUAD. Thus, we were the first to discover the high level of FTX in LUAD cell lines. Tumor growth and metastasis involve multiple cellular activities, such as proliferation, cell cycle, apoptosis, migration, invasion, and EMT [24, 25]. Herein, loss-of-function assay disclosed that silenced FTX inhibited cell proliferation by inducing cell cycle arrest and cell apoptosis, indicating the role of FTX in LUAD cell growth. Accordingly, we affirmed that FTX silence hindered LUAD tumorigenesis in vivo. Furthermore, FTX knockdown also repressed the metastasis-associated biological events in LUAD in vitro, including cell migration, invasion and EMT process in LUAD, and in vivo analysis confirmed that FTX1 deficiency decreased metastatic nodules. All results pointed out the oncogenic property of FTX in LUAD.

Recently, existing evidence has proposed a new regulatory mechanism that lncRNAs act as ceRNAs in the cytoplasm through competitively binding miRNAs to seclude their suppressive effect on target mRNAs [10]. Specifically, numerous miRNAs, participating in LUAD development, were reported to be regulated by lncRNAs [26-28]. FTX has been supported as a ceRNA in several tumors, such as hepatocellular carcinoma [29], osteosarcoma [30] and colorectal cancer [14]. Herein, our present study first showed that FTX was a cytoplasmic RNA and interacted with miR-335-5p in LUAD. Prior studies demonstrated that miR-335-5p was a tumor suppressor and involved in ceRNA network in renal cell carcinoma [31], gastric cancer [32] and osteosarcoma [33]. Here, miR335-5p was lowly expressed in LUAD cell lines and could combine with FTX. In addition, the inhibitory role of down-regulated FTX in LUAD cell progression could be antagonized by miR-335-5p inhibition. In conclusion, our research expounded that FTX was a molecular sponge of miR-335-5p.

Nucleobindin 2 (NUCB2) was featured as active regulators in the biological movements [34, 35]. In colorectal cancer, up-regulation of NUCB2 was reported to associate with metastasis and aggravate the carcinoma progression [36]. Additionally, NUCB2 was up-regulated in prostate cancer and served as a prognostic biomarker [37]. Our data first reported that NUCB2 was a downstream target of miR-335-5p and high NUCB2 expression was detected in LUAD cell lines. Functional assay conformed that NUCB2 played the oncogenic role in LUAD. Rescue experiments suggested that NUCB2 overexpression could counter repressive effect of silenced FTX on LUAD progression. Additionally, previous studies showed that NUCB2 can regulate several pathways, including AKT/mTOR and STAT3 pathway [19, 20]. Herein, we found that NUCB2 axis could affect AKT/ mTOR pathway by changing Ser473 (AKT phosphorylation site) and Ser2448 (mTOR phosphorylation site) but cannot affect STAT3 pathway. Although a study showed that UNCB2 affected both p-mTOR and p-STAT3 in hepatic gluconeogenesis [20], our data indicated that in NUCB2 cannot jointly regulate STAT3 and mTOR signaling in LUAD cells, probably because that in different cell types, the mechanisms and links between pathways and regulatory molecules vary. However, importantly, AKT/ mTOR pathway is widely recognized as a pathway regulating cell growth and metastasis [38, 39], so it is reasonable to deduce that $\mathrm{AKT} / \mathrm{mTOR}$ might be a downstream signaling for FTX/miR-335-5p/NUCB2 in LUAD. The detailed mechanism will be explored in the future for better explanation.

\section{Conclusion}

Conclusively, lncRNA FTX was an oncogene in LUAD and acted as a ceRNA to drive LUAD progression by targeting miR-335-5p/NUCB2 axis and mediating AKT/ mTOR pathway, providing a helpful theoretic basis to explore potential therapeutic strategies for the patients suffered from LUAD. Although we have explored AKT/ mTOR pathway in our study, further exploration on the downstream signals will be conducted in the future. 


\section{Supplementary information}

Supplementary information accompanies this paper at https://doi. org/10.1186/s12935-020-1130-5.

Additional file 1: Figure S1. (A) Cell cycle was analyzed with the transfec tion of sh-FTX or sh-NC. (B) The apoptosis of LUAD cells in sh-FTX or sh-NC group. (C) The proliferation of $\mathrm{H} 1650$ cells in sh-FTX, sh-FTX + miR-335-5p inhibitor or sh-NC group. (D) The apoptosis of LUAD cells in sh-FTX, shFTX + miR-335-5p inhibitor or sh-NC group. (E) The apoptosis of LUAD cells in sh-NUCB2 or sh-NC group. (F) The protein levels of $p-A K T, A K T$, p-mTOR, mTOR, p-STAT3, STAT3 in sh-FTX, sh-FTX + pcDNA3.1/NUCB2 or sh-NC group was detected. (G) The analysis of cell cycle in sh-FTX, shFTX + pcDNA3.1/NUCB2 or sh-NC group. $(H)$ The apoptosis of LUAD cells in sh-FTX, sh-FTX + pcDNA3.1/NUCB2 or sh-NC group. ${ }^{* *} \mathrm{P}<0.01$.

Additional file 2: Figure S2. (A) Images of tumors removed from the mice transplanted with sh-FTX or sh-NC transfected H1650 cells. (B-C) Tumor volume and tumor weight in sh-FTX or sh-NC groups were quantified. (D) Expression of FTX under the transfection of sh-FTX was determined by RT-qPCR. (E) HE staining was used to confirm the metastatic lung nodules. (F) NUCB2 protein level was evaluated after silencing of FTX using western blot assay. ${ }^{* *} \mathrm{P}<0.01$.

\section{Abbreviations}

IncRNA: Long noncoding RNA; LUAD: Lung adenocarcinoma; FTX: FTX transcript, XIST regulator; miR-335-5p: microRNA-335-5p; NUCB2: Nucleobindin 2; SCLC: Small cell lung carcinoma; NSCLC: Non-small cell lung carcinoma; ceRNA: Competing endogenous RNA; miRNA: microRNA; mRNA: Messenger RNA; DMEM: Dulbecco's Modified Eagle's Medium; FBS: Fetal bovine serum; RTqPCR: Reverse transcription quantitative polymerase chain reaction; GAPDH: Glyceraldehyde-3-phosphate dehydrogenase; shRNA: Short hairpin RNA; NC: Negative control; EdU: 5-Ethynyl-2'-deoxyuridine; SDS-PAGE: Sodium dodecyl sulfate-polyacrylamide gel electrophoresis; PVDF: Polyvinylidene fluoride; RIP. RNA immunoprecipitation; IgG: Immunoglobulin G; SD: Standard deviation; ANOVA: One-way analysis of variance; RISC: RNA-induced silencing complex; EMT: Epithelial-mesenchymal transition; FISH: Fluorescent in situ hybridization.

\section{Acknowledgements}

We thank all those who participated in this study.

\section{Authors' contributions}

$\mathrm{XH}$ and $\mathrm{HW}$ wrote the article, $\mathrm{BH}$ and LW devoted to the data, KY, JW, LW and HW contributed to the analysis, experiment and investigation. All authors read and approved the final manuscript.

\section{Funding}

Scientific research project of Tianjin Education Commission (natural science) (2018KJ073)

\section{Availability of data and materials}

Not applicable.

\section{Ethics approval and consent to participate}

We were used for animal experiments with the ethical approval of the the Second Hospital of Tianjin Medical University.

\section{Consent for publication}

Authors involved in this paper all signed written consent for publishing in your journal.

\section{Competing interests}

The authors declare that they have no competing interests.

\section{Author details}

${ }^{1}$ Department of Oncology, The Second Hospital of Tianjin Medical University, No. 23 Pingjiang Road, Hexi District, Tianjin 300211, China. ${ }^{2}$ Pain Management Center, The Second Hospital of Tianjin Medical University, Tianjin 300211, China. ${ }^{3}$ Central Laboratory/Tianjin Research Institute of Urology, The Second Hospital of Tianjin Medical University, Tianjin 300211, China.
Received: 30 October 2019 Accepted: 29 January 2020

Published online: 23 March 2020

\section{References}

1. Mao Y, Yang D, He J, Krasna MJ. Epidemiology of lung cancer. Surg Oncol Clin N Am. 2016;25(3):439-45.

2. Sun Q, Jiang CW, Tan ZH, Hou LY, Dong H, Liu K, Sun G, Liu YJ, Wang YQ, Lu $X C$, et al. MiR-222 promotes proliferation, migration and invasion of lung adenocarcinoma cells by targeting ETS1. Eur Rev Med Pharmacol Sci. 2017;21(10):2385-91.

3. Liu X, Wang J, Chen M, Liu S, Yu X, Wen F. Combining data from TCGA and GEO databases and reverse transcription quantitative $P C R$ validation to identify gene prognostic markers in lung cancer. OncoTargets Ther. 2019;12:709-20.

4. Quinn JJ, Chang HY. Unique features of long non-coding RNA biogenesis and function. Nat Rev Genet. 2016;17(1):47-62.

5. Zhu P, Wang Y, Huang G, Ye B, Liu B, Wu J, Du Y, He L, Fan Z. Inc-beta-Catm elicits EZH2-dependent beta-catenin stabilization and sustains liver CSC self-renewal. Nat Struct Mol Biol. 2016;23(7):631-9.

6. Berthenet K, Bokhari A, Lagrange A, Marcion G, Boudesco C, Causse S, De Thonel A, Svrcek M, Goloudina AR, Dumont S, et al. HSP110 promotes colorectal cancer growth through STAT3 activation. Oncogene. 2017;36(16):2328-36

7. Wen X, Liu X, Mao YP, Yang XJ, Wang YQ, Zhang PP, Lei Y, Hong XH, He QM, Ma J, et al. Long non-coding RNA DANCR stabilizes HIF-1alpha and promotes metastasis by interacting with NF90/NF45 complex in nasopharyngeal carcinoma. Theranostics. 2018;8(20):5676-89.

8. Dong J, Teng F, Guo W, Yang J, Ding G, Fu Z. IncRNA SNHG8 Promotes the Tumorigenesis and Metastasis by Sponging miR-149-5p and Predicts Tumor Recurrence in Hepatocellular Carcinoma. Cell Physiol Biochem. 2018;51(5):2262-74.

9. Tay Y, Kats L, Salmena L, Weiss D, Tan SM, Ala U, Karreth F, Poliseno L, Provero P, Di Cunto F, et al. Coding-independent regulation of the tumor suppressor PTEN by competing endogenous mRNAs. Cell. 2011;147(2):344-57.

10. Salmena L, Poliseno L, Tay Y, Kats L, Pandolfi PP. A ceRNA hypothesis: the Rosetta Stone of a hidden RNA language? Cell. 2011;146(3):353-8.

11. Rui $X, X u Y$, Jiang $X$, Ye W, Huang $Y$, Jiang J. Long non-coding RNA C5orf66-AS1 promotes cell proliferation in cervical cancer by targeting miR-637/RING1 axis. Cell Death Dis. 2018:9(12):1175.

12. Chen J, Huang X, Wang W, Xie H, Li J, Hu Z, Zheng Z, Li H, Teng L. LnCRNA CDKN2BAS predicts poor prognosis in patients with hepatocellular carcinoma and promotes metastasis via the miR-153-5p/ARHGAP18 signaling axis. Aging. 2018;10(11):3371-81.

13. Zhang W, Bi Y, Li J, Peng F, Li H, Li C, Wang L, Ren F, Xie C, Wang P, et al. Long noncoding RNA FTX is upregulated in gliomas and promotes proliferation and invasion of glioma cells by negatively regulating miR-342-3p. Lab Investig J Tech Methods Pathol. 2017;97(4):447-57.

14. Yang Y, Zhang J, Chen X, Xu X, Cao G, Li H, Wu T. LncRNA FTX sponges miR-215 and inhibits phosphorylation of vimentin for promoting colorectal cancer progression. Gene Ther. 2018;25(5):321-30.

15. Liu B, Pan S, Xiao Y, Liu Q, Xu J, Jia L. Correction to: LINC01296/miR-26a/ GALNT3 axis contributes to colorectal cancer progression by regulating O-glycosylated MUC1 via PI3K/AKT pathway. J Exp Clin Cancer Res CR. 2019;38(1):367.

16. Ji L, Li X, Zhou Z, Zheng Z, Jin L, Jiang F. LINC01413/hnRNP-K/ZEB1 axis accelerates cell proliferation and EMT in colorectal cancer via inducing YAP1/TAZ1 translocation. Mol Ther Nucleic Acids. 2019;19:546-61.

17. Feng S, Liu W, Bai X, Pan W, Jia Z, Zhang S, Zhu Y, Tan W. LncRNACTS promotes metastasis and epithelial-to-mesenchymal transition through regulating miR-505/ZEB2 axis in cervical cancer. Cancer Lett. 2019:465:105-17.

18. Yu C, Li L, Xie F, Guo S, Liu F, Dong N, Wang Y. LncRNA TUG1 sponges miR-204-5p to promote osteoblast differentiation through upregulating Runx2 in aortic valve calcification. Cardiovasc Res. 2018;114(1):168-79.

19. Yang M, Zhang Z, Wang C, Li K, Li S, Boden G, Li L, Yang G. Nesfatin-1 action in the brain increases insulin sensitivity through Akt/AMPK/TORC2 pathway in diet-induced insulin resistance. Diabetes. 2012;61(8):1959-68. 
20. Wu D, Yang M, Chen Y, Jia Y, Ma ZA, Boden G, Li L, Yang G. Hypothalamic nesfatin-1/NUCB2 knockdown augments hepatic gluconeogenesis that is correlated with inhibition of mTOR-STAT3 signaling pathway in rats. Diabetes. 2014;63(4):1234-47.

21. Wang C, Gu Y, Zhang E, Zhang K, Qin N, Dai J, Zhu M, Liu J, Xie K, Jiang Y, et al. A cancer-testis non-coding RNA LIN28B-AS1 activates driver gene LIN28B by interacting with IGF2BP1 in lung adenocarcinoma. Oncogene. 2019;38(10):1611-24.

22. Xiong DD, Li ZY, Liang L, He RQ, Ma FC, Luo DZ, Hu XH, Chen G. The LncRNA NEAT1 accelerates lung adenocarcinoma deterioration and binds to Mir-193a-3p as a competitive endogenous RNA. Cell Physiol Biochem. 2018;48(3):905-18.

23. Lu X, Huang C, He X, Liu X, Ji J, Zhang E, Wang W, Guo R. A novel long non-coding RNA, SOX21-AS1, indicates a poor prognosis and promotes lung adenocarcinoma proliferation. Cell Physiol Biochem. 2017:42(5):1857-69.

24. Sun Q, Li J, Li F, Li H, Bei S, Zhang X, Feng L. LnCRNA LOXL1-AS1 facilitates the tumorigenesis and stemness of gastric carcinoma via regulation of miR-708-5p/USF1 pathway. Cell Prolif. 2019;52(6):e12687.

25. Zhang R, Liu Y, Liu H, Chen W, Fan HN, Zhang J, Zhu JS. The long noncoding RNA SNHG12 promotes gastric cancer by activating the phosphatidylinositol 3-kinase/AKT pathway. Aging. 2019;11(23):10902-22.

26. Zhang ZW, Chen JJ, Xia SH, Zhao H, Yang JB, Zhang H, He B, Jiao J, Zhan BT, Sun CC. Long intergenic non-protein coding RNA 319 aggravates lung adenocarcinoma carcinogenesis by modulating miR-450b-5p/EZH2. Gene. 2018:650:60-7.

27. Yang L, Luo P, Song Q, Fei X. DNMT1/miR-200a/GOLM1 signaling pathway regulates lung adenocarcinoma cells proliferation. Biomed Pharmacother Biomedecine Pharmacotherapie. 2018;99:839-47.

28. Liu L, Liu L, Lu S. IncRNA H19 promotes viability and epithelial-mesenchymal transition of lung adenocarcinoma cells by targeting miR-29b-3p and modifying STAT3. Int J Oncol. 2019;54(3):929-41.

29. Liu F, Yuan JH, Huang JF, Yang F, Wang TT, Ma JZ, Zhang L, Zhou CC, Wang F, Yu J, et al. Long noncoding RNA FTX inhibits hepatocellular carcinoma proliferation and metastasis by binding MCM2 and miR-374a. Oncogene. 2016;35(41):5422-34.

30. Huang S, Zhu X, Ke Y, Xiao D, Liang C, Chen J, Chang Y. LncRNA FTX inhibition restrains osteosarcoma proliferation and migration via modulating miR-320a/TXNRD1. Cancer Biol Ther. 2020. https://doi.org/10.1080/15384 047.2019.1702405.
31. Wang K, Jin W, Song Y, Fei X. LncRNA RP11-436H11.5, functioning as a competitive endogenous RNA, upregulates BCL-W expression by sponging miR-335-5p and promotes proliferation and invasion in renal cell carcinoma. Mol Cancer. 2017;16(1):166.

32. Zhang LL, Zhang LF, Guo XH, Zhang DZ, Yang F, Fan YY. Downregulation of miR-335-5p by long noncoding RNA ZEB1-AS1 in gastric cancer promotes tumor proliferation and invasion. DNA Cell Biol. 2018;37(1):46-52.

33. Wang Y, Yang T, Zhang Z, Lu M, Zhao W, Zeng X, Zhang W. Long noncoding RNA TUG1 promotes migration and invasion by acting as a ceRNA of miR-335-5p in osteosarcoma cells. Cancer Sci. 2017;108(5):859-67.

34. Seon $\mathrm{S}$, Jeon $\mathrm{D}, \mathrm{Kim} \mathrm{H}$, Chung $\mathrm{Y}$, Choi $\mathrm{N}$, Yang $\mathrm{H}$. Testosterone regulates NUCB2 mRNA expression in male mouse hypothalamus and pituitary gland. Dev Reprod. 2017;21(1):71-8.

35. Xu H, Li W, Qi K, Zhou J, Gu M, Wang Z. A novel function of NUCB2 in promoting the development and invasion of renal cell carcinoma. Oncol Lett. 2018;15(2):2425-30.

36. Xie J, Chen L, Chen W. High NUCB2 expression level is associated with metastasis and may promote tumor progression in colorectal cancer. Oncol Lett. 2018;15(6):9188-94.

37. Zhang H, Qi C, Li L, Luo F, Xu Y. Clinical significance of NUCB2 mRNA expression in prostate cancer. J Exp Clin Cancer Res CR. 2013;32(1):56.

38. Zhang M, Liu S, Chua MS, Li H, Luo D, Wang S, Zhang S, Sun C, Han B. Correction: SOCS5 inhibition induces autophagy to impair metastasis in hepatocellular carcinoma cells via the PI3K Akt/mTOR pathway. Cell Death Dis. 2019;10(11):799.

39. Zhu MX, Wei CY, Zhang PF, Gao DM, Chen J, Zhao Y, Dong SS, Liu BB. Correction to: elevated TRIP13 drives the AKT/mTOR pathway to induce the progression of hepatocellular carcinoma via interacting with ACTN4. J Exp Clin Cancer Res CR. 2019;38(1):443.

\section{Publisher's Note}

Springer Nature remains neutral with regard to jurisdictional claims in published maps and institutional affiliations.
Ready to submit your research? Choose BMC and benefit from:

- fast, convenient online submission

- thorough peer review by experienced researchers in your field

- rapid publication on acceptance

- support for research data, including large and complex data types

- gold Open Access which fosters wider collaboration and increased citations

- maximum visibility for your research: over $100 \mathrm{M}$ website views per year

At BMC, research is always in progress.

Learn more biomedcentral.com/submissions 